\title{
Clergé et économie au Québec : historiographie et nouveaux problèmes
}

\section{Antonio Lechasseur}

Volume 46, 1979

URI : https://id.erudit.org/iderudit/1007114ar

DOI : https://doi.org/10.7202/1007114ar

Aller au sommaire du numéro

Éditeur(s)

Les Éditions Historia Ecclesiæ Catholicæ Canadensis Inc.

ISSN

0318-6172 (imprimé)

1927-7067 (numérique)

Découvrir la revue

Citer cet article

Lechasseur, A. (1979). Clergé et économie au Québec : historiographie et nouveaux problèmes. Sessions d'étude - Société canadienne d'histoire de l'Église catholique, 46, 45-59. https://doi.org/10.7202/1007114ar

Tous droits réservés @ Les Éditions Historia Ecclesiæ Catholicæ Canadensis Inc., 1979
Ce document est protégé par la loi sur le droit d'auteur. L'utilisation des services d'Érudit (y compris la reproduction) est assujettie à sa politique d'utilisation que vous pouvez consulter en ligne.

https://apropos.erudit.org/fr/usagers/politique-dutilisation/ 


\section{Clergé et économie au Québec : historiographie et nouveaux problèmes ${ }^{1}$}

L'histoire de l'Église catholique au Québec commence à profiter des enseignements de l'histoire économique et sociale telle que pratiquée depuis une dizaine d'années par une génération nouvelle d'historiens. On a longtemps étudié l'activité de l'Église et de son clergé sous l'angle des idéologies. Ce type de recherches est nécessaire pour bien comprendre quels sont les objectifs de l'Église et quelles orientations les penseurs catholiques ont données à l'action du clergé et à toute la société québécoise, l'Église étant une entreprise qui prend d'abord ses racines dans le monde des idées. Mais l'aspect matériel du pouvoir clérical au Québec a presque totalement été laissé de côté. L'objectif de ce texte est double : d'abord justifier l'étude du clergé en rapport avec les diverses facettes de l'économie et montrer ensuite comment certains historiens ont abordé cette question.

\section{LES BASES MATÉRIELLES DU POUVOIR CLÉRICAL AU QUÉBEC}

Avec la Révolution tranquille et depuis le début de la sécularisation de la société québécoise, les historiens commencent à cerner la réalité des vastes pouvoirs de l'Église catholique et l'ampleur du contrôle qu'elle a exercé sur la société aux XIX" et $\mathrm{XX}^{\prime \prime}$ siècles. La religion et l'Église catholique sont des constantes de l'histoire du Québec. Jcan-Charles Falardeau écrivait en 1952 que «la société canadienne-française a été, depuis les débuts mêmes de son établissement, à tel point circonscrite, contenuc et dominée tout entière par le clergé et les chefs ecclésiastiques, que son histoire se confond

1 Version remaniée d'une communication présentée au Congrès annuel de la Société canadienne d'Histoire de l'Église catholique, tenu au Cegep de Gaspé les 14 et 15 septembre 1979. Ce texte est largement inspiré du premier chapitre de mon mémoire de maîtrise : Propriété foncière 't clergé : Rimouski, 188/-1911, Montréal, M.A. (histoire), Université du Québec à Montréal, 1979, 170pp. 
en tout point avec celle de l'Église canadienne ${ }^{2} \gg$. C'est là un jugement peut-têre excessif mais qui suffit à illustrer comment l'Église catholique s'identifie à l'histoire du Québec.

Dans cette histoire, il y eut des périodes plus importantes que d'autres. La période 1867-1960 est une de celles-là. C'est au tournant du $X^{\prime \prime}$ siècle que l'Église catholique atteindra l'apogée de sa puissance. Ainsi, on a pu parler pour les années 1900 d'une Église "conquérante», et pour celles qui suivent, d'une Église «triomphaliste $^{3}$ \%. Le nouveau système politique confédératif donne au clergé une très grande autonomie à tel point que l'Église «devient rapidement, vers la fin du siècle, un élément tout à fait indispensable dans la vie sociale québécoise. Dans une certaine mesure, elle paraît davantage présente que l'État: par son réseau de paroisses et de diocèses qui quadrille l'espace habité, par le caractère social essentiel des institutions et des services qu'elle contrôle ${ }^{4}$ ». C'est par l'augmentation de ses effectifs et de son encadrement que l'Église deviendra cette force sociale de premier plan. On ne peut passer en revue tous les aspects de la domination du clergé sur la société québécoise. Toutefois, ces quelques remarques suffisent à justifier l'analyse du phénomène dans toute son ampleur.

Dans la perspective d'une histoire de l'Église, l'approche externe me semble plus riche dans la mesure où cela permet de définir le clergé comme un groupe social, c'est-à-dire appartenant à une structure sociale particulière. Plusieurs recherches menées jusqu ïci nous ont habitué à une vision monolithique de l'Église catholique et de son clergé. Les travaux récents tendent à montrer une Église où les paliers hiérarchiques sont nombreux et auxquels se rattachent des types différents de comportement, des intérêts communs, des privilèges et une reconnaissance sociale particulière. Le clergé urbain ne vit et ne pense pas de la même façon que le clergé de campagne.

De quelle manière faut-il envisager la situation et la pratique sociale du clergé? Peut-on affirmer que le clergé représente une classe sociale ou plus simplement une fraction de classe? Il y a trop de différences entre ses membres pour pouvoir classer tout le

2 Jean-Charles Falardeau. "Rôle et importance de l'Église au Canada français ». Esprit. 193-194 (août 1952), p. 214.

3 Nive Voisine et al., Histoire de l'Église catholique an Quéhec, 1608 1970. Montréal. Fides. 1971, pp. 39-72.

4 Paul-André Linteau. René Durocher et Jean-Claude Robert, Histoire du Quéhec contemporain. De la confédération à la crise (1867-1929), Montréal. Boréal Express. 1979, p. 234. 
monde sous une même étiquette. Le clergé se présente donc comme constitué de divers groupes susceptibles d'appartenir, selon leurs intérêts et leur idéologie, à l'une ou l'autre des classes sociales. Dans cette perspective, il s'agit de fractions de classe. C'est là une question importante qui sous-tend toute recherche sur le clergé québécois. Toute explication du phénomène clérical ne doit pas l'esquiver.

Les domaines principaux de l'action du clergé tiennent. en plus de la production de biens symboliques spirituels, au secteur des services, de l'éducation à l'hospitalisation en passant par la charité publique. Cette omniprésence a permis au clergé du Québec d'exercer un contrôle et un monopole idéologique quasi-unique. Ce pouvoir clérical dépassait de beaucoup la morale individuelle pour s'étendre aux divers aspects du comportement collectif. Ce large contrôle idéologique du clergé sur les individus et sur les groupes se devait de reposer sur des bases solides. Théoriquement, l'action de l'Église catholique s'est appuyée sur des assises matérielles robustes. Cela peut être considéré comme une lapalissade, mais si on pose le problème à l'inverse, il est peu probable que l'Église ait pu devenir une force dominante de la société si elle n'avait pas reposé, à la base, sur une structure économique lui permettant de s'imposer. Les intérêts spirituels et politiques ne sont pas à dédaigner dans la mesure où il faut invoquer l'interaction des facteurs pour expliquer le développement de l'Église catholique au Québec. mais les facteurs économiques sont de tout premier ordre.

En fait. l'étude de l'action économique du clergé permet d'identifier ou d'analyser celui-ci à titre de groupe social. Ellc permet de voir comment l'aspect matériel oriente son action et quels sont les rapports et les communautés que cela crée avec d'autres groupes ou d'autres classes sociales.

Les structures et les activités économiques de clergé ne sont pas partout et au même moment identiques. Son implication dans l'économic dépasse largement les besoins du groupe pour devenir dans certains cas une fin en soi. Ainsi, le clergé ne se limite pas au rôle qu'il s'est lui-même attribué. Dans ce cas, une étude uniquement idéologique laisse dans l'ombre plusieurs aspects importants.

Il est un autre point à soulever dans une analyse du clergé : il semble que c'est parfois sur des bases matérielles que se tissent des alliances qu'il contracte avec les groupes sociaux dominants, bien que la correspondance de leurs intérêts soit en bonne partie tributaire de la conjoncture. Ainsi, on comprend mieux comment 
l'Église catholique s'est accommodée de la plupart des régimes économiques et politiques.

Doit-on insister davantage pour montrer jusqu'à quel point lanalyse des bases économiques des groupes sociaux - et c'est peut-être plus difficile à justifier pour le clergé - est utile à l'histoire sociale du Québec?

Cela admis, il faut maintenant préciser un autre aspect du problème de l'analyse des bases matérielles du clergé québécois. Il s'agit de distinguer entre sa structure économique et ses activités proprement économiques. Ainsi, on ne peut mettre sur le même pied l'administration interne des communautés religieuses, par exemple. et les activités qui ont un rayonnement et une influence à l'échelle plus vaste de la communauté locale ou nationale. Ces aspects ont à peine retenu l'attention des chercheurs. Un ouvrage récent reconnâ̂t cette lacune en précisant les principaux licux d'intervention du clergé :

(...) le clergé a un rôle particulièrement important et il met volontiers son influence au service des grands entrepreneurs. Il y a d'ailleurs des hiérarchies à établir au sein du clergé. Le curé de paroisse a une influence considérable mais qui ne dépasse guère le niveau local. Les évêchés et les grandes communautés religieuses ont souvent à leur tête des individus issus de la bourgeoisic. Ces institutions disposent de ressources financières importantes, accumulées aul cours des décennies et qui échappent généralement à la taxation. Elles investissent dans des entreprises. elles agissent comme des institutions de crédit et de financement et même comme des quasi-banques dans les régions où le système bancaire est peu implanté. À ce niveau. les intérêts des grandes institutions religicuses sont assez près de ceux de la bourgeoisic. Il faut reconnaitre toutefois que cette dimension économique du pouvoir clérical nous est encore peu connuc. Le clergé peut d'ailleurs profiter d'une main-d'œuvre abondante et peu coûteuse: cette armée de frères et de sœurs qui sont recrutés parmi les familles nombreuses du monde rural 5 .

Donc, l'activité du clergé déborde le cadre interne de ses institutions pour accéder à un rôle et à une influence aux divers nivealux des structures économiques et sociales. Linteau, Durocher et Robert constatent le vide historiographique entourant cette question. À la limite. on pourrait affirmer qu'il n'y a vraiment pas eu au Québec

5 Ihid.. p. 175. 
jusqu'à maintenant un ouvrage ou une thèse qui s'est donné comme objectif premier de déblayer le terrain dans ce sens. Au moment de la discussion de l'historiographie on verra que les auteurs n'ont fait qu'aborder dans une autre perspective cet aspect de l'histoire du Québec. Ce sont principalement William F. Ryan, Normand Séguin et Brian Young.

À la lumière de ces remarques. la logique de lhypothèse se précise : il sagit de voir si en tant que groupe social le clergé a joué un rôle économique prépondérant. Ce rôle économique était pour lui une quasi-nécessité dans la mesure où il voulait conserver l'acquis et continuer à être une force sociale de premier plan. Ainsi, le clergé a été amené à simmiscer dans plusieurs des rouages de l'économic québécoisc. En d’autres termes, son pouvoir idéologique reposait sur de solides bases matérielles.

Il faut maintenant faire intervenir d'autres variables dans l'analyse des activités économiques de l'Église catholique au Québec: l'espace choisi pour étude, son état de développement économique et les domaines de son activité. À chacun de ces points on peut rattacher des sous-hypothèses, lesquelles serviront à guider la recherche vers l'objectif fixé.

Le choix du lieu dans lequel on souhaite faire cette recherche pèse tout au long du cheminement et en particulier sur les résultats qu'on obticnt. Si on retient pour étude une métropole, une capitale, une petite ville de province ou un village, il est clair qu'a priori le problème se présente différemment. Dans l'un ou l'autre cas, les questions suivantes surgissent: quelle; sont leurs physionomies sociales et économiques? comment se dessinent les rapports de production qu'on y trouve ? les rapports qu'ils entretiennent ? lesquels sont dominants et dominés? Enfin, à quel type de clergé a-t-on affaire? En somme, il s'agit de distinguer entre l'ensemble et les parties. C'est en faisant le lien entre tous ces facteurs que la recherche prend son sens.

Il importe d'identifier les secteurs économiques qui retiennent les faveurs du clergé. Le texte de Linteau, Durocher et Robert, précédemment cité, parle d'interventions dans les domaines de l'industrie (les services aux entrepreneurs, etc.), de la finance et du crédit. Ces types d'interventions directes dans l'économic caractérisent les hautes sphères de la hiérarchie et nécessitent l'immobi- 
lisation de sommes relativement importantes .Cela suppose une longue période d'accumulation reliée à des sources de revenus aussi efficaces que constantes. De plus, le clergé a bénéficié d’une maind'œuvre à bon marché qui lui était fournie par les campagnes québécoises aux prises avec diimportants surplus démographiques à la fin du XIX" siècle.

À mon avis, il est d'autres sources de revenus. Certaines à court terme relèvent des subventions de l'État dans le cadre des activités d'éducation et d'hospitalisation, des dons, des quêtes, etc. À plus long terme le clergé a certainement profité d'une vaste implication dans le domaine de la propriété foncière.

La propriété foncière est une donnée tout à fait primordiale pour l'analyse des structures économiques du XIX" siècle québécois. C'est un secteur dans lequel le clergé a été impliqué dès le début de la colonie française en Amérique :

En Nouvelle-France. l'Église avait reçu en même temps que la tâche de prêcher les valeurs religieuses aux colons et aux aborigènes. le monopole de léducation ct de l'hospitalisation. Pour lui permettre de remplir ces fonctions. on lui concède de riches seigneuries dont les revenus suffisaient à pourvoir les écoles. les collèges. les hôpitaux et les églises. et à assurer la subsistance des enseignants. du personnel hospitalier et des clercs : grâce à ce șstème. le clergé séculier et les ordres religieux étaient devenus les plus riches propriétaires fonciers de la colonie et le groupe de pression le plus influent dans les affaires politiques 6 .

De là l'hypothèse suivante: la propriété foncière a constitué un champ d'intervention économique privilćgié pour le clergé des villes du Québec. Reste à s'entendre sur les types d'intervention. Sagit-il de la gestion d'un patrimoine hérité d'un autre âge. ou bien est-ce un secteur dans lequel le clergé investit et spécule? Des différences se manifestent sûrement selon les caractères de la localité ou de la société dans laquelle se trouve le clergé en question. D'ores et déjà. on peut supposer que la propriété foncière n'est pas un secteur passif d'intervention économique et que dans certains cas elle est devenue une source de revenu considérable. Il est même possible

6 Alfred Dublc. "Les classes sociales au Canadas. Annules. É(onomie's. Socicités. Civilisations, 22. + (juillet-août 1967), p. 835. 
que le clergé, selon les circonstances, ait soumis ou soustrait de vastes espaces au développement urbain capitaliste. C'est une hypothèse qui mérite d'être posée. Globalement, l'implication du clergé au plan du capital foncier soulève la délicate question de sa position de classe.

L'historiographie a toujours considéré l'Église catholique comme une force sociale conservatrice alignée sur les traditions et garante d'un certain consensus. Cette vision de l'élite cléricale tend à être nuancée par les recherches récentes.

Si le clergé a été impliqué de façon significative dans l'économie, notamment dans le secteur de la propriété foncière, il est probable que les intérêts sociaux traditionnels et les intérêts économiques cléricaux ne coïncidèrent pas toujours. Le haut-clergé, de par son activité foncière et la composition de son personnel bureaucratique. ne s'apparente-t-il pas à la bourgeoisie, elle-même très active dans le domaine foncier? On considérera à titre d'hypothèse que le clergé québécois joue sur plusieurs tableaux à la fin du XIX $\mathrm{X}^{\mathrm{e}}$ siècle, à tel point que ses comportements économiques et sociaux peuvent éclairer sa position de classe, notamment la similitude entre intérêts cléricaux et bourgeois au plan du capital foncier. Ainsi, il profite du développement économique alors qu'il a souvent été présenté comme hostile au progrès libéral. La thèse de William $F$. Ryan est significative à cet égard. Bien sûr, l'étude d'autres secteurs d'intervention économique pourrait préciser davantage cet aspect de la position de classe du clergé. L'étude de la propriété foncière et l'étude des rapports et des communautés d'intérêts ainsi créés suffit pour mettre sur la piste d'une nouvelle compréhension de la réalité du pouvoir clérical au Québec à la fin du XIX" siècle. Éventuellement, il faudra voir comment les intérêts économiques cléricaux et bourgeois tendent à s'exprimer au plan politique. Il y a donc nécessité de sortir de l'étude strictement idéologique pour comprendre comment l'exercice de pouvoirs, quels qu'ils soient, est ancré dans la réalité matérielle. Poser le problème pour l'ensemble du Québec demanderait plusieurs années de recherche. Des études de cas pourront peut-être répondre partiellement aux questions que soulève l'hypothèse développée ici. Il faut maintenant se demander en quoi cette conception des liens entre clergé et économie se rapproche et se distingue des travaux de l'historiographie contemporaine. 


\section{LES RAPPORTS CLERGÉ ET ÉCONOMIE DANS L'HISTORIOGRAPHIE}

Au Québec et au Canada, les recherches d'histoire sur les liens entre l'économie et le clergé ne sont pas très nombreuses: on les compte sur les doigts d'une seule main. Seuls S.D. Clark, William F. Ryan, Normand Séguin et Brian Young ont abordé l'une ou l'autre des questions que ces rapports soulèvent.

\section{Éthiques Rel.igIeuses et ÉCONOMIE : S.D. Clark}

Le sociologue Samuel Delbert Clark a été l'un des premiers au Canada à relier développement économique et phénomènes religieux. Après la seconde guerre mondiale, il publie deux articles qui touchent à ce problème : "The Religious Sect in Canadian Economic Development 6:1 ». Ces travaux font référence à ceux de Max Weber et de Kurt Samuelson qui ont voulu relier le développement de l'éthique protestante et celui du capitalisme. En somme, pour Clark, le facteur religieux a été au Canada une force de maintien ou de solidarité de la communauté et «a force of dominant importance ${ }^{7}$ 》. Dès les débuts de la Nouvelle-France, l'Église exerce un contrôle théocratique sur la société coloniale alors qu'après la Conquête, elle trouve un nouveau support dans son alliance avec l'État.

L'approche de Clark tend à montrer que les Églises, particulièrement l'Église catholique au Québec, ont constitué des freins au développement économique. Voici de quelle façon :

In the first place, the heavy drain upon the material resources of the country in maintaining institutions of religious worship has weakened the economic energies of the population.

[...]

Where a large share of the capital and labour of the society has been diverted to support of religious establishments the problem of securing a sufficient surplus to promote economic growth has been considerably intensified ${ }^{8}$.

6:1 Publiés respectivement dans Journal of Economic History. VII (Supplement, 1947). pp. 89-103 et dans Canadian Journal of Economics and Political Science, XII (Nov. 1946). pp. 439-453, et repris tous deux dans The Developing Canadian Community. Toronto, University of Toronto Press, 1962, pp. 147-182.

7 Op. cit., p. 169.

$8 \quad$ Ibid., p. 172. 
Il considère que ce fut le cas au Canada français :

In French Canada heavy ecclesiastical taxation in one form or another has meant that the capital required for the establishment of industry has come to a considerable extent either from outside capitalist groups, from the state, or from the church itself 9 .

Somme toute, l'auteur croit que l'éthique religieuse catholique rend difficile l'exercice de l'éthique capitaliste. Comme notre connaissance de l'histoire du Québec a bien progressé depuis la parution de cet article, il faut se demander si la thèse de Clark est vraiment valable. Contre elle, on peut dire que l'argent investi dans de grandes constructions a élé remis en circulation par le clergé. De plus, le clergé a certainement fait des investissements dans divers secteurs de l'économie, soit par l'achat d'obligations de compagnies ou de l'État. Mais pour Clark, le problème se pose au niveau du modelage des mentalités économiques. À ce sujet, les avis sont partagés puisque pour d'autres, il semble n'y avoir «no necessary inconsistency between 'the present day Roman Catholic ethic' and 'the economic development ethos' $10 »$. Ce problème est encore loin d'être tranché.

\section{Ci.frgé et dévfloppement économiQue: William F. Ryan}

L'ouvrage de William F. Ryan, The Clergy and Economic Growth in Quebec, 1896-1914, est la plus importante tentative pour lier l'Église catholique à la croissance économique du Québec à la fin du XIX" siècle et au début du XX". En fait, Ryan se demande "in what direction did the Church throw her weight during this period of economic growth ${ }^{11}$ ». En étudiant les régions du Saguenay et de la Mauricie, il montre que de 1896 à 1914, il s'est produit des changements majeurs dans chacune de ces deux régions et analyse la part qui en revient au clergé.

Ryan étudie longuement le rôle du curé de paroisse dans ces régions au moment où plusieurs entreprises anglo-saxonnes s'y installent pour l'électricité, la coupe du bois, le papier, etc. Les curés

9 Ihid., p. 173.

10 William F. Ryan, Clergy and Economic Growth in Quebec, 189619/4, Québec, Les Presses de l'Université Laval, 1966, p. 300. Il cite ici Mario Zanartu, Roman Catholic Ethic and Economic Development, Thèse de Ph.D., Université Columbia, Faculté des sciences politiques, 1962, p. 260.

11 Ibid., pp. 6-7. 
de ces nouvelles paroisses jouèrent un grand rôle dans ces développements en plus de voir à l'organisation de la paroisse comme telle, à la construction d'une église ou l'aménagement d'écoles. Le curé devient un intermédiaire et un modérateur entre les compagnies et les travailleurs, non seulement en cas de conflits ou de grèves mais à longueur d'année. Comme Ryan le dit dans le cas de la Laurentide Pulp Company Limited de Grand-Mère, le curé fut "the 'unofficial' arbitrator between the company and the men ${ }^{12}$ ", au point de rendre les affaires de l'entreprise plus prospères.

Le rôle des évêques y est également discuté du fait de leur attitude favorable à ces nouveaux investissements. Ils se feront les propagandistes de l'idéologie agriculturiste, les défenseurs des Caisses populaires et les fondateurs des premiers syndicats catholiques. Ces interventions eurent des effets positifs ou négatifs sur l'économie. Pour Ryan, la plupart d'entre elles contribuèrent à créer un climat bénéfique à l'industrialisation. Les évêques entretinrent toujours de bonnes relations avec les chefs d'entreprises capitalistes qui se propagèrent un peu partout en Mauricie et au Lac Saint-Jean.

De pliss. William F. Ryan discute longuement de l'activité du clergé dans le système d'éducation au Québec. À ce niveau, l'Église ne s'est toujours adaptée que tardivement aux réalités du monde moderne et aux développements de l'économie. Il faudra attendre la deuxième décennie du $X^{\prime \prime}$ " siècle pour voir naître les premières écoles spécialisées. Malgré les efforts du clergé et des commissions scolaires, le degré de scolarisation populaire restera très faible pendant longtemps.

L'auteur conclut que l'implication du clergé dans le domaine économique était fonction de l'association qu'il faisait alors entre la nation. la religion et l'économie. Les évêques encouragent et favorisent la colonisation dans le but de contrer l'émigration des Québécois aux États-Unis tout en demeurant les défenseurs de la prospérité de leur cité et de ses industries. Comme l'évêque, le curé tend lui aussi "to identify himself with the needs, hopes and aims of his parish ${ }^{13} »$.

Ryan conclut que le rôle de l'Église dans le développement économique a été positif sans pour cela être déterminant. Sur les

12 Ibid.. p. 66.

13 Ibid.. p. 296. 
points abordés, cette conclusion est presque irréfutable. Mais, en analysant l'influence qu'exerce le clergé par le biais de l'éthique prônée, Ryan ne dévoile qu'une partie du tableau.

Enfin, on pourrait qualifier l'ouvrage de Ryan de tentative libérale d'explication de l'histoire économique du Québec. Son approche semble dénuée de critiques et les questions qu'il pose jettent assez peu de lumière sur le sens véritable de l'implication du clergé dans l'économie des nouvelles régions qui naissent au Québec à la fin du XIX siècle. En réalité, cette activité ne faisait qu'accentuer la dépendance de la majorité paysanne et ouvrière au développement du capitalisme. Si le clergé espérait de cette façon conserver sa propre emprise sur les classes ouvrières, il livrait par contre cette masse rendue docile aux griffes de l'entrepreneur capitaliste anglo-saxon ou canadien-français. Donc, l'auteur laisse dans le noir toute cette question de l'appartenance de classe du clergé et du sens de ses positions sociales en tant que groupe distinct. La concordance des intérêts du clergé avec ceux des entrepreneurs capitalistes ne soulève aucun problème chez Ryan. Encore faudrait-il qu'il se soit posé la question.

\section{ASPECTS MATÉRIFLS DU POUVOIR CLÉRICAL RURAL : NORMAND SÉGUIN}

Parlons maintenant d'un ouvrage récent en histoire économique et sociale qui a soulevé des discussions et qui a posé des problèmes nouveaux aux historiens du Québec. Il s'agit du livre de Normand Séguin: La conquête du sol au 19" siècle. L'auteur y étudie le mouvement de colonisation qu'a connu le Saguenay Lac Saint-Jean au XIX" siècle à l'aide du cas de Notre-Dame d'Hébertville. Il inscrit sa recherche dans la problématique du développement du sousdéveloppement dans le but de "démontrer que la colonisation du Saguenay s'explique fondamentalement par les relations de dépendance qui tiennent une agriculture de subsistance à la remorque d'activités forestières ${ }^{14} \gg$. En reprenant la thèse de William F. Ryan sur la collaboration clérico-capitaliste dans le développement du Saguenay, Séguin va plus loin que son prédécesseur en abordant la question des bases matérielles du pouvoir clérical dans une société qu'on a longtemps qualifiée de traditionnelle. Voilà ce qui fait pour moi l'intérêt et l'aspect stimulant de cet ouvrage.

14 Normand Sf́guin. La conquĉte du sol au 19" siècle. Trois-Rivières, Éditions du Boréal Express, 1977. p. 23. 
Séguin étudie successivement "les bases matérielles du pouvoir clérical dans la communauté hébertvilloise du XIX" siècle», "le contexte des relations du curé avec ses ouailles», et enfin "l'école et le contrôle clérical dans la paroisse ». Dans le cadre de ce texte, je n'aborderai que le premier de ces aspects.

À Hébertville au XIX" siècle, le curé et la fabrique ont les revenus les plus élevés : "le clergé paroissial contrôle des revenus ordinaires nettement supérieurs - entre 50 et $100 \%$ généralement à ceux des municipalités de la paroisse et du village et de la commission scolaire réunis 15 ». Dans plusieurs circonstances, les paroissiens doivent supporter le coût d'entretien et de construction des édifices religieux. On prélève des revenus cxtraordinaires par des répartitions et des quêtes spéciales. Tous ces prélèvements permettent au curé de devenir l'homme le plus puissant de la communauté. Sa puissance est renforcée par le droit qu'il a de poursuivre toute personne fautive ou en retard dans ses paiements et corvées. "Il est évident qu'il existe un rapport étroit entre les revenus qui confirment la puissance matérielle et la reconnaissance de l'Église consacrée dans les statuts ${ }^{16}$ », dira Normand Séguin. En fait, «les questions d'argent occupent une place très importante dans les relations du curé avec ses paroissiens 17 ». Comme la fabrique est hautement impliquée dans le commerce des grains au niveau local, du fait que les dîmes sont le plus souvent payées en nature, Séguin montre clairement que l' "organisation religieuse dans la paroisse rurale au XIX" siècle est une des premières instances économiques de la communauté 18 ».

Séguin ne cherche pas à creuser cette question de façon particulière. Il n'en reste pas moins que ses conclusions sur cet aspect du pouvoir clérical se rapprochent de très près de l'hypothèse émisc en première partie à l'effet que cet immense pouvoir du clergé, aux $\mathrm{XIX}^{\prime}$ et $\mathrm{XX}$ " siècles, prend ses racines dans des réalités bien matérielles. L'économie d'une localité rurale est bien influencée par le curé et la fabrique. Séguin a donc contribué à soulever le voile qui dissimule les rapports entre clergé et économie. La connaissance du phénomène a fait un grand pas depuis l'ouvrage de Ryan.

15 Ibid., p. 189.

16 Ihid.. p. 193.

17 Ihid., p. 194.

18 Ibid., p. 196. 


\section{Le Clergé entrepreneur: La thèse de Brian Young}

La thèse de Brian Young, Promoters and Politicians ${ }^{19}$, sur les entreprises de chemin de fer de la Rive Nord du Saint-Laurent de 1854 à 1885, traite d'un aspect longtemps soupçonné mais rarement confirmé de l'histoire de l'Église catholique au Québec : le clergé entrepreneur.

Dès 1857 , lors des premières discussions en vue de la construction du chemin de fer de la Rive Nord, le clergé se montre intéressé à une participation financière. Ainsi, le Séminaire de Québec promet $£ 300$ et l'archevêque de Québec, $£ 400$. Brian Young cite une lettre très intéressante du vicaire-général de Québec, adressée aux diverses communautés religieuses, qui illustre comment le clergé défendait, à cette occasion, ses intérêts économiques :

Le soussigné ... déclare qu'il approuvera volontiers la détermination que celles-ci pourraient prendre de contribuer à la construction du dit chemin de fer. Ce projet intéresse à un haut point les Corporations qui possèdent des propriétés tant dans la ville de Québec que sur le parcours de la voie ferrée. et leur souscription libérale en cette circonstance les rendra extrêmement populaires dans l'opinion publique. C'est dans ce sens que Nos Seigneurs l'Archevêque de Québec et l'Évêque de Tloa s'en sont expliqués avant leur départ. Les Dames religieuses savent peut-être aussi que $\mathrm{M}^{\mathrm{wr}}$ a pris pour $£ 100$ d'actions 20.

Ce premier projet n'eut pas les suites escomptées. Il faut attendre la Confédération pour que le Montreal Colonization Railway gagne plusieurs appuis politiques. Le support clérical ne manque pas de se faire sentir. Le soutien moral était un élément tout aussi important aux yeux des promoteurs et cela permettait d'espérer de rondelettes subventions des deux paliers de gouvernement. L'évêque de Montréal, un pcu comme celui de Québec, voyait d'un très bon œil la multiplication des réseaux de chemin de fer.

La participation financière du clergé visait bien plus que le développement de nouvelles régions et le bien-être de leurs habitants. Le clergé avait compris que le salut spirituel tant prêché passait

19 Brian J. Young, Promoters and Politicians. The North-Shore Railways in the History of Quchec, 1854-1885, Toronto, University of Toronto Press, 1978, 193pp.

20 Cité ibid., p. 16. 
d'abord par la santé matérielle du corps clérical et de ses ouailles. De plus, ce type d'intervention économique illustre jusquà quel point il existait une concordance d'intérêts entre les politiciens et le clergé du fait de leur appui mutuel. À cet effet, l'exemple de la famille Langevin est éclairant. Les questions d'argent occupent une large place dans leurs rapports. Le chemin de fer. comme il avait le pouvoir de dessiner et d'orienter le développement urbain et rural, représentait pour le clergé une excellente occasion pour s'intégrer. survivre et profiter du «progrès »:

The higher clergy and seminarians were also attracted to railways through their concern to choose the best site for a hospital or school and their interest in the value of church properties. Their vigourous participation in community life made it impossible to ignore railway development. In Quebec City the Ursulines sisters donated a terminus to the Quebec and Gosford Railway, in the St. Maurice Valley new parish and diocesan boundary were formed in accordance with traffic flow along railway lines. and in Montreal the diocese inherited shares in the Joliette Railway 21.

En somme, l'épisode de la construction ferroviaire est un exemple de l'implication du clergé dans l'économie. On se doute bien que l'investissement de capitaux dans diverses entreprises ait pu tenter le clergé. Ce n'est là probablement qu'un chapitre de l'histoire du clergé comme entrepreneur. Aurait-il détenu des actions dans d'autres entreprises? À l'époque, il devait y avoir des investissements aussi rentables que les chemins de fer. Le clergé n’a pas manqué de faire fructifier ses «talents».

Voilà quelques exemples qui illustrent le bien-fondé de l'analyse des rapports entre le clergé et l'économie dans un sens large. On distingue nettement deux tendances de la recherche dans ce domaine : les deux premiers auteurs - Clark et Ryan - abordent le problème sous l'angle difficile des mentalités ou des influences économiques d'une éthique religieuse. Par contre, Séguin et Young traitent directement de l'implication du clergé dans l'économie. Ces deux façons sont intéressantes et complémentaires, mais la seconde me semble porteuse d'un nouvel élan qui concorde avec les progrès de l'histoire économique et sociale.

21 Ibid., p. 36. 
Il importe donc de porter une attention particulière aux divers types d'intervention économique du clergé. Les travaux devraient se multiplier dans ce domaine. À ma connaissance, aucune recherche n'a exploité exclusivement cet aspect de l'histoire de l'Église catholique au Québec. B. Young et N. Séguin ne l'ont fait que partiellement lors d'études portant sur d'autres problèmes. Il faudrait connaître le degré et le type d'implication économique des divers groupes cléricaux tels les évêchés, les communautés d'hommes et de femmes, les fabriques et les clercs comme individus, tant dans les milieux comme Montréal et Québec qu'en province. On connaît très peu de choses des activités économiques du Séminaire de Québec, des Sulpiciens à Montréal et des importantes communautés religieuses aux $\mathrm{XIX}^{\mathrm{e}}$ et $\mathrm{XX}^{\mathrm{e}}$ siècles. Personneilement, j'ai entrepris d'étudier l'implication et les activités foncières du clergé à Rimouski de 1881 à $1911^{22}$. La multiplication des monographies pourra éclairer ce problème important qu'est l'étude des assises matérielles du clergé pour l'histoire du comportement des groupes sociaux au Québec.

Antonio LeCHasseur, Université du Québec à Montréal.

22 Voir supra, note 1. 